\title{
La inteligencia emocional desarrolla colaboradores exitosos en las organizaciones
}

\author{
Emotional intelligence develops successful collaborators in the \\ organizations
}

\begin{abstract}
Washington Edy Santillán Marroquín. ', María Cristina Araque Salazar. ${ }^{2}$, Verónica Elizabeth Villarroel Jácome. ${ }^{3}$ \& Tania Magdalena López Villegas. ${ }^{4}$
\end{abstract}

Recibido: 12-09-2021 / Revisado: 17-09-2021 /Aceptado: 22-10-2021/ Publicado: 05-11-2021

\begin{abstract}
DOI: https://doi.org/10.33262/concienciadigital.v4i4.1.1926

Every day we found more evidence than it allows us to confirm that a correct academic formation based on the psychological area that it includes, management of the emotions that go from the childhood, aid greatly to develop emotional intelligence like "the capacity to adapt to the different scenes from the life". Objective, to analyze the influence that has emotional intelligence in the labor performance of the collaborators/workers within the organizations and knowledge in what emotional level is to manage to harness the performance level. Methodology, one treats to apply descriptive, bibliographical and reflective a methodology, that would help employees of a company to mobilize and to fortify the emotions to prover in them their development, plan of race and systemic formation. On the other hand, the application of the empathy would be due to foment where we recognize the emotions of the others to be able to include/understand the different situations that cross our next ones (family, friends, fellow workers or related others). Results, conscience in the modern companies for

\footnotetext{
${ }^{1}$ Docente de posgrado de psiquiatría de la Universidad Tecnológica Equinoccial UTE. Coordinador Académico del Instituto Superior Tecnológico Bernardo O'Higgins, wsantillan1@gmail.com ORCID: https://orcid.org/0000-0003-1980- 6832, Quito- Ecuador.

2 Docente del Instituto Superior Tecnológico Bernardo O’Higgins, mcass2008@ hotmail.com ORCID: https://orcid.org/0000-0002-8425- 7289, Quito- Ecuador.

3 Coordinadora de Bienestar Estudiantil del Instituto Superior Tecnológico Bernardo O'Higgins, bienestar@instituto-ohiggins.com ORCID: https://orcid.org/0000-0002-6291- 505X, Quito- Ecuador.

${ }^{4}$ Docente del Instituto Superior Tecnológico Bernardo O'Higgins, tmlopezvi@yahoo.es ORCID: https://orcid.org/0000-0002-5736- 2550, Quito- Ecuador.
} 
recruitment and contract collaborator with a "great dose" of emotional intelligence for one better coexistence and then they can fit with the diversity that exists at the present time on subjects: generational, cultural, sort, politician, social, religious, etc., this without a doubt will hit in the enterprise culture, productivity and yield. It was demonstrated, that the qualification programs and formation in several workers increased to a $25 \%$ the average of emotional intelligence and with this the social abilities a $50 \%$. Finally, it was demonstrated that $65 \%$ of the labor success must to a good use of emotional intelligence, not only with itself, but with the others.

Keywords: emotions, intelligence, empathy, adaptation, formation, competitions, communication.

\section{Resumen}

Cada día encontramos más evidencia que nos permite confirmar que una correcta formación académica fundamentada en el área psicológica que incluye, gestión de las emociones que van desde la niñez, ayuda grandemente a desarrollar la inteligencia emocional como "la capacidad de adaptarse a los diferentes escenarios de la vida". Objetivo, analizar la influencia que tiene la inteligencia emocional en el desempeño laboral de los colaboradores/trabajadores dentro de las organizaciones y saber en qué nivel emocional se encuentran para lograr potenciar el nivel de desempeño. Metodología, se trata de aplicar una metodología descriptiva, bibliográfica y reflexiva, que ayudarían a los trabajadores de una empresa a movilizar y fortalecer las emociones para proveer en ellos su desarrollo, plan de carrera y formación sistémica. Por otro lado, se debería fomentar la aplicación de la empatía donde reconocemos las emociones de los demás para poder comprender las distintas situaciones que atraviesan nuestros próximos (familia, amigos, compañeros de trabajo u otros relacionados). Resultados, concienciar en las empresas modernas para que tengan y contraten colaboradores con una "gran dosis" de inteligencia emocional para una mejor convivencia y que puedan encajar con la diversidad que existe en la actualidad sobre temas: generacionales, culturales, género, político, social, religioso, etc., esto sin duda impactará en la cultura empresarial, productividad y rentabilidad. Se evidenció, que los programas de capacitación y formación en varios trabajadores incremento en un $25 \%$ el promedio de la inteligencia emocional y con esto las habilidades sociales un 50\%. Finalmente, se demostró que el $65 \%$ del éxito laboral se debe a un buen uso de la inteligencia emocional, no sólo consigo mismo, sino con los demás.

Palabras Claves: emociones, inteligencia, empatía, adaptación, formación, competencias, comunicación.

\section{Introducción}

Existen varios estudios realizados que explican de una manera distinta el cómo ven otras personas el tema de la inteligencia emocional. 
En un estudio acerca del impacto de renovar un programa MBA se demostró que la IE de los estudiantes puede verse afectada por un programa educativo diseñado para desarrollar competencias en áreas más allá de lo cognitivo (Boyatzis y Saatcioglu, 2008), aunque los numerosos cambios en este programa no dejaron claro qué aspectos específicos incrementan la IE.

En otro estudio realizado por Cook-Sather (2012), con estudiantes universitarios, no se pudo evidenciar el incremento de la IE de los estudiantes de primero a cuarto, ni que dicho incremento sea superior en los de artes liberales respecto a los de contabilidad, al poder mejorar habilidades como la comunicación, el pensamiento crítico y el razonamiento ético (éstos tienen una IE superior tanto en primero como en cuarto). Pero sí pudieron demostrar que la IE de los estudiantes de contabilidad se incrementa más si el programa incluye contenidos no empresariales y más relacionados con las artes liberales.

La inteligencia emocional tuvo su primera aparición en el año 1990 por un artículo escrito por Peter Salovey y John Mayer, pero fue gracias a Daniel Goleman con su libro la inteligencia emocional escrito en el año 1999 que el concepto de inteligencia emocional se esparció rápidamente con éxito.

El Instituto Capgemini procedió a realizar una entrevista a 750 directivos, 1500 colaboradores y a su vez se pudo obtener información más detallada a 15 especialistas del campo académico con lo cual se determinó que:

La inteligencia emocional será una práctica necesaria en el futuro y quizás la demanda aumente hasta seis veces más en los siguientes cinco años. Una fuerza laboral emocionalmente inteligente beneficiaría tanto a las organizaciones como a los trabajadores.

Un estudio de McClelland (1998), menciona que, con datos de más de treinta organizaciones, encontró que "aquellas organizaciones con una amplia gama de competencias de IE relacionadas con la automotivación, la conciencia social y habilidades sociales, son mejores que la media, y concretamente superaron los objetivos anuales de ingresos entre un $15 \%$ y un $20 \%$ ".

Finalmente, podemos mencionar qué los beneficios que puede alcanzar una organización si se aplica en gran nivel la inteligencia emocional en los trabajadores, éstas tendrán talento humano mejor calificado, que optimizará el desempeño laboral, evitaría la rotación del personal y prevendrá los conflictos que generan desgaste y costes importantes.

\section{Metodología}

Según lo explica Gómez (2006) aplicará una metodología descriptiva, bibliográfica y reflexiva, que produce información de tipo cualitativo, en la cual se busca "especificar los beneficios en la formación de las personas, mientras más temprano mejor en inteligencia emocional", las características y los perfiles de personas, equipos de 
trabajo, comunidades, procesos, relaciones o cualquier otro fenómeno vinculado con el tema; es decir, miden, evalúan o recolectan datos sobre el impacto de la inteligencia emocional en generar ambientes de trabajo más satisfactorios que incidan trasversalmente sobre la productividad y rentabilidad de las empresas.

La metodología comprende un proceso de análisis que debe contener la información y los planteamientos, por ello Tamayo (2004) comienza por clarificar el tipo de investigación, estableciendo diferencias fundamentales entre las distintas variedades y de acuerdo al propósito, a los datos, a los conocimientos, los medios utilizados, etc.; es decir, se debe entender que al profundizar en una investigación científica uno de los puntos medulares es el que se encuentra contenido en la metodología, ya que es en el apartado donde se delinea la ruta a seguir en forma sistemática y ordenada, con la finalidad de realizar una planificación adecuada, en recursos y tiempo.

\section{Resultados}

Goleman (2001), define a la inteligencia emocional como la forma de interactuar con las demás personas, esta tiene en cuenta las emociones y la habilidad de controlar impulsos, autoconciencia, motivación, entusiasmo, perseverancia, empatía, entre otras que son indispensables para una excelente y creativa adaptación en interacción social.

Robbins y Judge (2009), mencionan que la inteligencia emocional es la capacidad que una persona posee para reconocer las emociones que experimenta y detectar las emociones de los demás, manejar claves e información emocional. Las personas que son capaces de conocer sus emociones y pueden detectar las de los demás, son más eficaces en sus trabajos. Varios estudios mencionan de manera determinante que la inteligencia emocional desempeña un rol importante en el desempeño en el trabajo.

La gestión inteligente de las emociones en los colaboradores de una empresa, lleva al éxito integral de las organizaciones, utilizando adecuadamente el autoconocimiento, la autorregulación, motivación, empatía y las habilidades sociales las cuales contienen otras competencias integradas como: autoconfianza, innovación, responsabilidad, compromiso, compresión, liderazgo entre otras.

Los resultados parten por recapitular las formas de clarificar la información que se encuentra contenida en las organizaciones, a través de las evidencias vivenciales de todos y cada uno de los trabajadores en su día a día de trabajo.

Las emociones, las emociones están siempre presentes desde el nacimiento de los seres humanos y juegan un papel relevante en la construcción de la personalidad, así como en las interacciones sociales.

Cuando se habla de un desarrollo sistémico de las personas, es importante tener en cuenta las capacidades cognitiva, física, lingüística, moral, así como afectiva y emocional. Las emociones intervienen en todos los procesos evolutivos y son la base de la toma de decisiones. 
La emoción, del latín Emovere, 'mover hacia', se considera según Alvarez et. al. (2000) como un estado complejo del organismo caracterizado por una excitación o perturbación que se genera como respuesta a un acontecimiento tanto externo como interno del individuo. Es una reacción psicofisiológica cuya función es adaptativa, cumpliendo un papel central en los procesos de autoprotección y autorregulación del organismo frente a diversas situaciones.

Para Bericat (2012), las emociones son complejas ya que no son respuestas automáticas que se dan en ciertos momentos sino por el contrario tiene un bagaje donde se conjugan las creencias y las convicciones propias de las personas y que son propias de la adaptación y de la propia evolución de la especie frente a los estímulos que presenta el entorno. En definitiva, las emociones son un reflejo de nosotros mismos y de nuestra vida por sobre todo de nuestra infancia, que se puede tener traumas que marcan cierto tipo de comportamientos y reacciones que deben ser reguladas desde la inteligencia y el autocontrol.

Importancia de las emociones, según Goleman (1999), las emociones son importantes porque influyen en nuestros pensamientos y viceversa, por lo cual frente a la emoción actuamos de una determinada manera y tomamos decisiones; por ejemplo, si estamos tristes podemos actuar bajo esa mirada un tanto desalentadora. También las emociones nos permiten comunicarnos y entender a las personas de nuestro entorno, poder identificar como se sienten y cómo actuar en las distintas interacciones, cuando podemos entenderlas estamos siendo también empáticos, compasivos y tolerantes con los demás.

Las emociones no bien gestionadas, afectan nuestra capacidad de razonamiento y claridad, se ha identificado que el sistema límbico es responsable de los procesos de aprendizaje y recuerdos y la amígdala es responsable de las alertas de las emociones; esta última, puede tener una conexión inmediata con el tálamo sin pasar por el neocórtex, como una vía rápida que le lleva a una interpretación y acción a la persona, que en ocasiones puede ayudar a su cuidado, sin embargo, en otras le puede ocasionar hacer interpretaciones que están basadas en situaciones anteriores (huella emocional) y que le hacen responder erróneamente, debido a que no hay un análisis completo o más profundo de la situación de ese momento (Goleman, 1999).

Otro aspecto interesante que lo plantean Martínez y Muñoz (2005), es que la amígdala de acuerdo a los estudios que se han realizado se desarrolla y madura en etapas tempranas del niño/a, a diferencia de lo que ocurre con otras zonas como las prefrontales del cerebro, por esta razón las vivencias de la infancia tienen alto impacto desde las emociones, cuando aún el niño/a no está en capacidad de expresarlo verbalmente o de analizar mejor la vivencia.

El autoconocimiento permite identificar las emociones que están presentes en un determinado momento y la posibilidad de observarse en una forma neutral y también controlar los impulsos (Plachta, 2011). 
Es importante identificar más allá de la inteligencia relacionada con el CI (coeficiente intelectual), que existen otras inteligencias como la intrapersonal e interpersonal, siendo la inteligencia social fundamental en afrontar la vida; por lo que las emociones marcan una diferencia en cuanto al éxito y bienestar de la persona (Goleman, 1999).

¿Para qué sirven las emociones? Goleman (2001), menciona que los sentimientos y anhelos más profundos son puntos de referencia necesarios en la especie humana y debe gran parte de la existencia de los asuntos humanos a los sentimientos, que son de más larga duración que las emociones. El poder de las emociones es extraordinario por ejemplo, sólo un amor potente puede llevar a unos padres a ir más allá de su propio instinto de supervivencia individual. Desde el punto de vista del intelecto, se trata de un sacrificio indiscutiblemente irracional, pero, visto desde el corazón, constituye la única elección posible.

Las emociones sirven para cubrir una necesidad, nos llevan a una acción; lo importante es notarlas en primer lugar y luego poderlas trasmitir y gestionar en forma adecuada en nuestro entorno para construir relaciones saludables y a largo plazo.

Las cinco emociones básicas son: alegría, afecto, enojo, tristeza, miedo, algunos autores también consideran el desagrado; estas, a su vez dan lugar a otras emociones en forma similar a lo que ocurre con los colores primarios; así la alegría puede dar lugar al optimismo, esperanza, fe. (Castro, 2013).

La emociones empiezan por una sensación en el cuerpo como calor, enrojecimiento, temblor, cosquilleo y estos aspectos fisiológicos no los podemos controlar, pues simplemente llegan a nosotros; lo que si podemos es gestionar y responder en forma adecuada a las mismas (Arroyo, 2012).

Otro aspecto relevante en cuanto a las emociones es que estas, sean funcionales, lo cual quiere decir, que se encuentren en balance y son saludables para nuestra vida; incluso emociones que consideramos agradables o positivas como el afecto en una condición disfuncional, puede afectar nuestro bienestar y salud emocional, por ejemplo el apego que estaría relacionado con la dependencia y el alejamiento de la autonomía personal, o en el caso de la alegría que al estar disfuncional podemos volvernos optimistas en exceso pero poco realistas o eufóricos (Muñoz, 2015).

Es relevante que haya un contacto con la necesidad que nos está trayendo la emoción para poder resolverla en forma beneficiosa.

Goleman (1999), considera que hay dos tipos de mentes, una racional y otra emocional, la cual está directamente relacionado con el sistema límbico específicamente con la amígdala.

Para gestionar las emociones es importante:

1. Reconocer las emociones que estoy experimentando sin juzgar.

2. Validar lo que siento. 
3. Utiliza técnicas de regulación emocional como la respiración, postura corporal y actividades de relajación.

4. Buscar apoyo en espacios de conversación en relación a como estoy, hablar con alguien de confianza que me facilite la expresión, aunque no solucione el problema.

5. Propiciar pensamientos positivos.

6. Alimentación saludable, ejercicios físicos, buen descanso y dormir lo suficiente, distraerse con actividades de recreación.

¿Qué son las emociones y los estados de ánimo?, Robbins y Judge (2009), explican que antes se deben analizar y aclarar tres términos que se entremezclan, el afecto, las emociones y el estado de ánimo.

El afecto cubre un rango amplio de sentimientos que experimentan las personas.

Es un concepto que agrupa emociones y estados de ánimo. Las emociones son sentimientos intensos a alguien o algo. Los estados de ánimo suelen ser menos intensos que las emociones y que con frecuencia carecen de un estímulo contextual.

La mayoría de expertos creen que las emociones son más fugaces que los estados de ánimo. Por ejemplo, si alguien trata a alguien con descortesía la persona se enojará. Ese sentimiento intenso de ira es probable que llegue y se vaya con rapidez, quizá en cuestión de segundos. Puede ser que cuando se encuentre de mal humor se pueda sentir mal por varias horas.

Las emociones se caracterizan por su intensidad hacia algo o alguien, los estados de ánimo son menos intensos y se instalan a partir de las emociones más recurrentes en nuestra historia personal, para identificarlos podemos hacernos la pregunta sobre cuáles son las emociones que están más presentes en nuestra vida (Robbins y Judge, 2009).

Los estados de ánimo están relacionados con nuestra forma de ver el mundo, así lo podemos observar como un lugar seguro y de ser así vamos a transitar contentos, optimistas confiados, o podemos verlo como un lugar amenazante y de inseguridad, tiene relación con la experiencia que hemos tenido frente a las distintas situaciones, con nuestras ideas y sistema de creencias (Plachta, 2011).

Inteligencia emocional en las organizaciones, una vez que conocemos lo que es la inteligencia emocional y definimos que son la gestión de las emociones, el siguiente paso es encontrar cómo se aplica la inteligencia emocional en las organizaciones.

Hoy en día las organizaciones deben estar a la vanguardia, adaptándose constantemente a procesos de cambio, los cual directa o indirectamente crea un desequilibrio en los hábitos y emociones de los trabajadores. Solo aquellas organizaciones que puedan adaptarse más fácilmente a dichos cambios podrán generar una verdadera ventaja sobre su competencia. 
En este sentido la inteligencia emocional (IE) cobra un protagonismo esencial para el manejo adecuado de las emociones de los trabajadores en la gestión del cambio.

Tomemos en cuenta que las emociones influyen directamente en el comportamiento del ser humano en cualquier ámbito en el cual sea participe. Ahora bien, si centramos nuestra atención específicamente en el ámbito organizacional, nos daremos cuenta que es de suma importancia el manejo adecuado de la inteligencia emocional tanto en un nivel individual, equipos de trabajo, ámbito social y organizacional.

De acuerdo a Martin (2018), la inteligencia emocional es la diferencia entre la intención tomando en cuenta la forma de desarrollar y gestionar las emociones con eficiencia. Así la inteligencia emocional es responsable de generar el diálogo que permite tener una vida de vinculación y crecimiento continuo con las personas que amas. Si hablas de una carrera profesional exitosa, la inteligencia emocional es igual a prepararse intelectualmente para llevar a cabo las metas, integrando dentro de si mismos los conocimientos y habilidades de interconexión y de conexión con quienes están en nuestro entorno en niveles de voluntad que suman hacia la excelencia en el trabajo.

Según Goleman \& Cherniss (2013), existe una influencia directa mutua entre la inteligencia emocional de un individuo y el grupo al cual pertenece. Es decir, tanto el individuo emocionalmente inteligente influye en la IE del grupo, como el grupo que profesa este tipo de inteligencia influye en el desarrollo de la IE de cada uno de sus integrantes. Y esta dinámica permite que la IE sea un factor importante en la eficacia, eficiencia y resultados de una organización.

La inteligencia emocional en el trabajo, ha existido desde siempre la controversia entre la importancia del CI (coeficiente intelectual) y la IE (inteligencia emocional) al momento de realizar una adecuada selección de personal que cubra adecuadamente los requisitos de algún puesto de trabajo que requiera la organización. Para ello se debe tomar en cuenta que, el hecho de que un trabajador cuente con la suficiente inteligencia cognitiva para ejercer un puesto de trabajo no significa ciertamente que este se convierta obligatoriamente un trabajador estrella o un futuro líder de entre sus compañeros, es necesario para ser un excelente trabajador contar con la inteligencia emocional.

En esta misma línea Robbins y Judge (2009), menciona que la IE desempeña un rol trascendental en el rendimiento y los resultados del ser humano en el trabajo, ya que algunas investigaciones muestran que aquellos trabajadores considerados como "estrellas" presentaban en común la cualidad de poder relacionarse mejor con los demás.

Sin lugar a duda la IE en los trabajadores permite generar un ambiente de trabajo más adecuado, en el cual todos los colaboradores experimentan una mejor calidad de su vida laboral y emocional, lo cual permite generar mayor productividad y mejores resultados cualitativos para la organización. 
En este sentido Biedma-Ferrer (2017), corrobora que existe una relación positiva entre la IE y el liderazgo, la motivación y el rendimiento; lo cual causa una relación directa consecuente con los resultados que se obtiene en el trabajo.

Además, para afianzar esta postura podemos mencionar que en una publicación realizada en el blog titulado Senior Manager, publicación de Pedro Rojas denomina:

"la felicidad en el trabajo es sinónimo de productividad" y para ello, se plantea nueve razones por las cuales el trabajador o colaborador debe sentirse a gusto en su lugar de trabajo, pues así sus acciones darán los efectos más positivos posibles:

- Un trabajador feliz, es un trabajador motivado y optimista.

- Un trabajador feliz desarrolla todo su talento y da mucho más de sí mismo.

- Un trabajador feliz se adapta mejor al equipo.

- Un trabajador feliz es mucho más creativo.

- Un trabajador feliz se adapta mejor a los cambios.

- Un trabajador feliz es menos propenso a equivocarse.

- Un trabajador feliz es un trabajador saludable y un promotor de seguridad en el trabajo.

- Un trabajador feliz resuelve problemas, no los crea.

- Un trabajador feliz es un buen discípulo de su líder.

Destacar las razones que provocan emociones positivas tornando más eficiente la labor del colaborador, ya sea por satisfacción con su entorno laboral o por sentirse auto motivado, es uno de los puntales de esta investigación.

La inteligencia emocional y los líderes, toda organización está conformada por una estructura compleja en la cual cada uno de los puestos de trabajo que la integran son importantes para que la organización se mantenga en gran nivel, independientemente de su organigrama.

Por consiguiente, en cualquier tipo de estructura organizacional todos los niveles jerárquicos existentes requieren el manejo de una IE adecuada que permita sinergia entre todos sus miembros para alcanzar los objetivos y las estrategias de negocio que la organización persigue.

Sin embargo para Valencia y Jerry (2012), existen casos como, los informáticos, los técnicos de laboratorio o los contadores no precisan de una marcada inteligencia emocional en su trabajo, aunque nunca está de más. La verdad es que a menudo se llega a los 50 años pensando en lo magnífico que habría sido madurar a los 30 (o incluso antes). Se puede entonces, sin más preámbulos, llegar a la conclusión de que a todas las personas les conviene mejorar en esta madurez inteligente que se denomina inteligencia emocional, y desde luego el mundo empresarial lo demanda visiblemente. Cabe ya preguntarse, para qué medir la inteligencia emocional, pero la respuesta está en la mente del lector: para saber en qué se debe mejorar sostenidamente en cada caso particular. 
Un ser humano emocionalmente bajo sería un ser humano incompleto. Particularmente, haciendo referencia a la importancia de la inteligencia emocional en el trabajo de los líderes directivos, se debe tomar como referencia, para identificar las prioridades en la mejora continua del desempeño, las competencias requeridas por el perfil del cargo.

Algún éxito anterior podría confundir a los directivos y a las personas en general sobre sus auténticos perfiles de competencias. Obviamente, los directivos inteligentes digieren bien sus éxitos y aun sus fracasos y deben estar conscientes de sus desaciertos para reaprender de ellos, sin desmotivarse.

Consecuentemente, toda organización requiere que sus colaboradores cohesionen sus habilidades trabajando conjuntamente en diversos niveles jerárquicos que establece la estructura organizacional, lo cual determina las funciones de cada uno de los puestos de trabajo. En este sentido los altos cargos gerenciales se encargan de vigilar el trabajo de sus subalternos y asumen una responsabilidad conjunta de las actividades realizadas de su equipo, de aquí, el problema principal radica que no todas las personas que ocupan altos cargos son líderes, ni todos los líderes ocupan un alto cargo en una organización (Chiavetano, 2009).

Por tal motivo, varios autores organizacionales coinciden en la idea de no confundir el término liderazgo con administración.

El liderazgo es un término mucho más trascendental, es un proceso de influencia directa con el equipo de trabajo, en el cual los seguidores reconocen libre y voluntariamente el poder que ejerce el líder sobre el equipo de trabajo.

Robbins y Judge (2009), define al liderazgo como "la aptitud para influir en un grupo hacia el logro de una visión o el establecimiento de metas.

Un verdadero líder, es aquel que tiene la habilidad de darse cuenta como se sienten sus colaboradores, de esta forma actuar oportunamente si identifica cierto grado de insatisfacción o desmotivación (Goleman \& Cherniss, 2013).

Es decir, la inteligencia emocional sirve para conocer nuestras propias emociones y entender lo que piensa y sienten los demás. Por tal motivo las organizaciones deben identificar aquellos trabajadores con un liderazgo potencial y formarlos para un posterior ascenso interno que les permita desarrollar sus habilidades de mando en beneficio de sus compañeros y de la organización en general.

Un verdadero líder organizacional debe manejar cinco componentes básicos de la inteligencia emocional:

1. El líder debe ser consciente de sus propios sentimientos, emociones y saber conocer y apreciar las de los demás. Tiene la habilidad de manejar sus emociones de manera sana y madura. 
2. Un verdadero líder sabe gestionar sus emociones de manera que escoge el medio más adecuado para canalizar sus emociones sin herir las susceptibilidades de quienes lo rodean.

3. Constantemente el líder encuentra una motivación personal que le permita afrontar con entusiasmo y optimismo cualquier adversidad que se le presente.

4. Posee la habilidad de sentir empatía con las personas que lo rodean, poniéndose en el lugar de ellos, comprendiendo los problemas y sentimientos ajenos.

5. Tiene capacidad de edificar relaciones sociales constructivas que permitan conectarse con los demás, influir en su equipo de tal manera que pueda convertirse en un ente conciliador que maneja desacuerdos y resuelve conflictos para alcanzar propósitos comunes.

Además, es importante mencionar que la IE en un líder le permitirá ser partícipe activo de su equipo y tener la habilidad de recibir críticas y reacciones negativas por parte de sus subalternos, permitiendo que esto sea una oportunidad de conocer el grado de satisfacción que tiene su equipo de trabajo y recopilar esta retroalimentación para realizar las debidas acciones para mejorar su gestión de liderazgo.

Resulta muy relevante el conocimiento de la inteligencia emocional para los líderes directivos y dirigentes a la hora de desarrollar e implementar las diferentes tareas que componen el proceso complejo de la dirección de talentos.

\section{Conclusiones}

- Es esencial que los directivos, gerentes, supervisores y líderes sean inteligentes emocionalmente, ya que enfrentan éxitos y fracasos, lo que lleva a que deben estar seguros de sus decisiones y motivarse cuando no ocurre lo planeado, tengan problemas o deban enfrentar situaciones complejas, ya que están a cargo de ellos y todo el personal pendientes de sus decisiones.

- Una persona satisfecha con gran inteligencia emocional y que sigue sus sueños, va a poder motivarse a sí mismo, ser optimista, desarrollar talentos esenciales como la comunicación, trabajo en equipo, liderazgo entre muchos otros talentos.

- Las metas que una persona tiene en la búsqueda de su bienestar y su estabilidad llega a estar muy relacionado con la organización en que trabaja, ya que en el caso de que esta incentive este plan de vida de los trabajadores se traduce en que va a dar todo su potencial y va a cumplir con los objetivos planteados.

- La inteligencia emocional se relaciona directamente con el rendimiento de los trabajadores en la organización a la cual pertenecen, a su vez, parte de un proceso consciente de entendimiento e introspección que exige un trabajo interno que permita desarrollar un hábito muy bien estructurado de reacciones.

- La empatía es una de las principales características de un líder con inteligencia emocional, permitiéndole de esta forma ponerse en el lugar de las personas que conforman su equipo, convirtiéndose en un ente conciliador ante cualquier tipo de conflicto interno que pudiese suscitar. 
Finalmente, tras conocer lo que es la inteligencia emocional, el rol de las emociones y cómo todo esto se desenvuelve en nuestras organizaciones, podemos llegar a concluir que con un buen manejo y gestión emocional; además, con un sólido autoconocimiento y ser empáticos, se puede llegar al bienestar integral, que se traduce al final del día, como el éxito sistémico en la organización.

\section{Referencias bibliográficas}

Álvarez, M., Bisquerra, R., Fita, E., Martínez, F., \& Pérez, N. (2000). Evaluación de programas de educación emocional. Revista de investigación educativa, 18(2), $587-599$.

Arroyo, A (2012). Habilidades gerenciales: Desarrollo de destrezas, Competencias y actitud (1. ${ }^{\mathrm{a}}$ ed.). Colombia, Bogotá. Ecoe Ediciones.

Bericat Alastuey, E. (2012). Emociones. Sociopedia. isa, 1-13.

Biedma-Ferrer, J. M. (2017). La inteligencia emocional en las organizaciones y su impacto sobre algunos aspectos de los Recursos Humanos.

Boyatzis, R. E., \& Saatcioglu, A. (2008). A 20-year view of trying to develop emotional, social and cognitive intelligence competencies in graduate management education. Journal of management development.

Castro, M. D. C. (2013). Coaching En Accion Herramientas De Intervencion (1. a ed.). México, México D.F. Editorial Trillas.

Chiavetano, A. (2009). Comportamiento organizacional. La dinámica del éxito en las organizaciones. Segunda edición México DF: Mc Graw Hill

Cook-Sather, A. (2012). Amplifying Student Voices in Higher Education: Democratizing Teaching and Learning through Changing the Acoustic on a College Campus: La amplificación de las voces del alumnado en la Educación Superior: democratización de la enseñanza y el aprendizaje en un centro universitario a través del cambio de su acústica. Ministerio de Educación.

Goleman Daniel, (1999). La inteligencia emocional en la empresa, Javier Vergara, editor, Buenos Aires.

Goleman, D. (2001). La Práctica de la Inteligencia Emocional. Barcelona España: Editorial Kairós.

Goleman, D., \& Cherniss, C. (2013). Inteligencia emocional en el trabajo: cómo seleccionar y mejorar la inteligencia emocional en individuos, grupos y organizaciones. Editorial Kairós. 
Gómez, M. (2006). Introducción a la metodología de la investigación científica. Córdova. Argentina: Bruja

Martín, E. M. A. (2018). Inteligencia emocional. Editorial Elearning, SL.

Martínez, R. M., \& Muñoz, S. R. (2005). La inteligencia emocional y su relación con el proceso directivo en el contexto empresarial. Ingeniería Industrial, 26(1), 18-24.

McClelland, D. C. (1998). Identifying competencies with behavioral-event interviews. Psychological science, 9(5), 331-339.

Muñoz, M. (2015). Los coho modos de relación o interacción (primera reimpresión), México, Coyoacán. IHPG.

Plachta, G.(2011), PNL Mapas para el cambio (1. ${ }^{\mathrm{a}}$ ed.). Argentina, Buenos Aires. Editorial Albatros Saci.

Robbins, S. y Judge, T. (2009) Comportamiento Organizacional., Décimo Tercera Edición. Pearson. México

Tamayo, M. (2004). El proceso de la investigación científica. Editorial Limusa.

Valencia, G. F., \& Jerry, I. S. (2012). La inteligencia emocional en el éxito empresarial. Abya-Yala, UPS. Quito

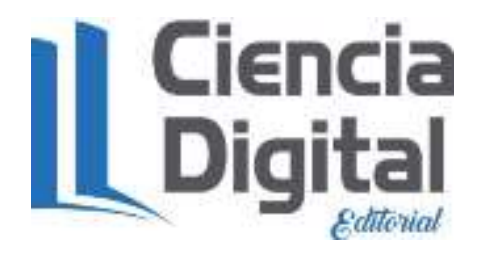




\section{PARA CITAR EL ARTÍCULO INDEXADO.}

Santillán Marroquín, W. E., Araque Salazar, M. C., Villarroel Jácome, V. E., \& López Villegas, T. M. (2021). La inteligencia emocional desarrolla colaboradores exitosos en las organizaciones. $\quad$ ConcienciaDigital, $\quad 4(4.1), \quad 67-80$. https://doi.org/10.33262/concienciadigital.v4i4.1.1926

\section{LCiencia}

El artículo que se publica es de exclusiva responsabilidad de los autores y no necesariamente reflejan el pensamiento de la Revista Conciencia Digital.

El artículo queda en propiedad de la revista y, por tanto, su publicación parcial y/o total en otro medio tiene que ser autorizado por el director de la Revista Conciencia Digital.

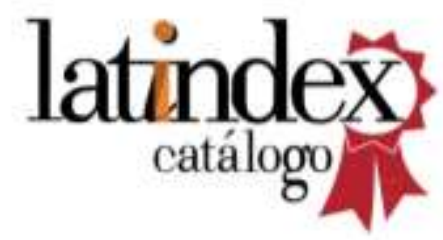

